\title{
Soft Radio-Frequency Identification Sensors: Wireless Long-Range Strain Sensors Using Radio-Frequency Identification
}

\author{
Lijun Teng, ${ }^{1, *}$ Kewen Pan, ${ }^{2, *}$ Markus P. Nemitz, ${ }^{1,3}$ Rui Song, ${ }^{1}$ Zhirun $\mathrm{Hu}^{2}$ and Adam A. Stokes ${ }^{1}$
}

\begin{abstract}
Increasing amounts of attention are being paid to the study of Soft Sensors and Soft Systems. Soft Robotic Systems require input from advances in the field of Soft Sensors. Soft sensors can help a soft robot to perceive and to act upon its immediate environment. The concept of integrating sensing capabilities into soft robotic systems is becoming increasingly important. One challenge is that most of the existing soft sensors have a requirement to be hardwired to power supplies or external data processing equipment. This requirement hinders the ability of a system designer to integrate soft sensors into soft robotic systems. In this article, we design, fabricate, and characterize a new soft sensor, which benefits from a combination of radio-frequency identification (RFID) tag design and microfluidic sensor fabrication technologies. We designed this sensor using the working principle of an RFID transporter antenna, but one whose resonant frequency changes in response to an applied strain. This new microfluidic sensor is intrinsically stretchable and can be reversibly strained. This sensor is a passive and wireless device, and as such, it does not require a power supply and is capable of transporting data without a wired connection. This strain sensor is best understood as an RFID tag antenna; it shows a resonant frequency change from approximately 860 to $800 \mathrm{MHz}$ upon an applied strain change from $0 \%$ to $50 \%$. Within the operating frequency, the sensor shows a standoff reading range of $>7.5 \mathrm{~m}$ (at the resonant frequency). We characterize, experimentally, the electrical performance and the reliability of the fabrication process. We demonstrate a pneumatic soft robot that has four microfluidic sensors embedded in four of its legs, and we describe the implementation circuit to show that we can obtain movement information from the soft robot using our wireless soft sensors.
\end{abstract}

Keywords: soft sensing, RFID, antenna, wireless, passive

\section{Introduction}

\section{Soft sensors}

CofT SENSORS IS an application-driven research field and $\mathcal{W}$ has received much attention in recent decades. A soft sensor is a measuring tool that is made of a single polymer or a mixture of soft polymers. These soft polymers are usually softer than the materials they interact with, which make them inherently safe. One prominent example is electronic skin. Electronic skin is also called "second skin" referring to its softness; electronic skin is softer than human skin and makes it to an ideal substructure for sensor arrays. ${ }^{1}$ A close relative of the field Soft Sensors is the field of Soft Robotics. One motivating factor for using Soft Robotics is the fact that the materials of the robots are softer than the environments with which they interact, and this compliance makes them ideal for applications involving human-robot interaction. ${ }^{2-4}$

\footnotetext{
${ }^{1}$ The School of Engineering, Institute for Integrated Micro and Nano Systems, The University of Edinburgh, The King's Buildings, Edinburgh, United Kingdom.

${ }^{2}$ Microwave and Communication Systems Group, School of Electrical and Electronic Engineering, University of Manchester, Manchester, United Kingdom.

${ }^{3}$ Department of Computer Science and Engineering, University of Michigan, Ann Arbor, Michigan.

*This is a joint article and these authors contributed equally to this work.

(C) Lijun Teng et al. 2018; Published by Mary Ann Liebert, Inc. This Open Access article is distributed under the terms of the Creative Commons License (http://creativecommons.org/licenses/by/4.0), which permits unrestricted use, distribution, and reproduction in any medium, provided the original work is properly cited.
} 
Autonomous agents that perceive and act upon their immediate environment (robots) are influenced by sensor research. Sensor research impacts a robots' sensor modalities, sensor accuracy, or measurement range besides others. The concept of integrating sensing capabilities at low cost has also become increasingly important, especially for certain subgroups in robotics, namely soft robotics, modular robotics, and swarm robotics. In such cases, lower cost systems are motivated by design for manufacturability allowing one to commercialize or to scale a robot system. In the past decade, the community has started to make soft robotic actuators, such as (1) ionic actuators, ${ }^{5-7}$ (2) pneumatic actuators, ${ }^{8-11}$ or (3) dielectric actuators ${ }^{12-14}$ to just name a few. These actuators can benefit from soft sensors from feedback force control.

Kim et al. ${ }^{15}$ have proposed a stretchable sensor that is made of curvilinear silicon ribbons in an elastic substrate. This novel work allows high-precision complex active electronics to be densely integrated over a few square centimeters area while achieving high degrees of stretchability, bendability, and twistability. Park et al. ${ }^{16}$ designed a soft artificial skin with multiple layers of microscale liquid metal channels embedded in an elastomeric substrate to detect multiaxial strain and normal pressure. Single-core copper wire was used in the above researches to interconnect an external data-logger. Other research work, such as Tabatabai et al., ${ }^{17}$ Park et al., ${ }^{18}$ and Majidi et al. ${ }^{19}$ have also used similar connection methods.

Although the above-mentioned soft sensors show a promising future for stretchable electronics, they are active sensors that require a power supply and signal processing capabilities, such as a microcontroller with analog-digital converters. As reported in the study of Teng et al., ${ }^{20}$ each of these sensing systems has drawbacks in the interconnection to other devices, and these limitations hinder the uptake of the soft sensor devices into integrated systems. Embedding several such sensors in an untethered soft robotic system is difficult due to power consumption and practical considerations in wiring.

Stretchable antennas that have great potential to be used as wireless soft sensors have been reported in recent years. Stretchable liquid metal antennas have been presented by Cheng et al., ${ }^{21,22}$ Kubo et al., ${ }^{23}$ and So et al. ${ }^{24}$ Although these antennas have shown fascinating electronic and mechanic properties, they require coaxial radio frequency (RF) connector or hardwiring to connect to external data processing equipment, which limits their capability of being implemented in sensing applications such as movement monitoring.

Huang et al. ${ }^{25}$ mentioned the idea of integrating a measurement chip in stretchable antennas, but the researchers did not provide the solution of integration. Kim et al. ${ }^{26}$ demonstrated epidermal electronics using near-field communication; this wireless sensor is biocompatible and achieves sensor reading ranges of a few centimeters. Cheng and $\mathrm{Wu}^{27}$ also proposed a reversibly stretchable, large-area wireless strain sensor, which can work wirelessly $>5 \mathrm{~m}$ with a strain limitation of $15 \%$. These studies pave the way for making wireless stretchable antennas that can be used as soft sensors in soft robotic systems.

\section{Design of a new soft radio-frequency identification sensor}

In this article, we present the design, fabrication, and characterization of a long-range monitoring wireless strain sensor, which is capable of remotely detecting high tensile dynamic strain up to $50 \%$ at $>7.5 \mathrm{~m}$. This sensor benefits from a combination of radio-frequency identification (RFID) technology and microfluidic electronics fabrication technology. The proposed sensor is also a configurable RFID tag antenna. The RFID integrated circuit chip we integrated in the proposed sensor has very small dimensions $(0.4 \mathrm{~mm} \times 0.4 \mathrm{~mm} \times 0.2 \mathrm{~mm})$, so it will not affect the deformability of the sensor.

The electronic properties of this antenna are highly sensitive to mechanical strains at the frequency of operation (800$860 \mathrm{MHz}$ ). This sensor can withstand repeated mechanical strain while retaining electrical functionality and can return to its original state after stretching. In comparison to existing stretchable sensors, our sensor is a completely passive and wireless device. This RFID sensor can be embedded into soft robots without requiring any power consumption from the original robotic device. The feature of wireless power extends the lifetime of the device, removing the need for changing batteries, or integrating an energy harvesting system.

Additionally, most of existing gallium-based liquid alloy sensors were designed based on measuring the resistance change of the liquid metal, but the long-time stability of these sensors will be affected by the oxidation of gallium (gallium-based liquid alloy is easily oxidized). Our proposed RFID strain sensor was designed based on resonant frequency change upon strain. Benefiting from the high electrical conductivity of Galinstan, the skin depth (a measure of how closely electric current flows along the surface of a material) of Galinstan under $800-860 \mathrm{MHz}$ is only a few micrometers. Even though the Galinstan in the channels has a thin oxide layer at the surface, the oxide will not have a large effect on the resonant frequency of the sensor.

In this work, we describe the potential of integrating the proposed RFID sensor into soft robots, which have pneumatic actuators. Most of the existing soft sensors have a requirement to be hardwired to power supplies or to signal processing equipment. This dependence on electrical tethers limits the practical use of soft sensors in many promising applications in real-world conditions. Although, recently, researchers have presented some fully untethered soft robotic systems, ${ }^{28-31}$ most of them require bulky onboard components, such as batteries, microprocessors, pumps, or motors. These onboard components add additional weight on the soft systems, and thereby, the systems need to carry more powerful actuators.

In this work, we intended to develop untethered soft sensors, which can work wirelessly by dispensing, entirely, with the need for onboard power and processors. The proposed sensor can be fully embedded into soft robots without adding any other devices or additional electrical tethers, so that one can eliminate the wire and weight pressures on soft robotic systems. The relatively long measurement working range can allow the soft robots to move freely in a reasonably large area.

\section{Principles and Implementation}

\section{RFID technology}

RFID is a low-cost technology that allows the passive wireless communication of data. In recent decades, RFIDs have been used in many projects such as in robot motion 
sensors, ${ }^{32}$ indoor localization, ${ }^{33}$ and distributed sensor networks. ${ }^{34}$ An RFID system usually consists of a transponder (sometimes called RFID tag) and the reader. The reader transmits electromagnetic waves to reach out to the RFID tag; if the RFID tag is within the operating range, it utilizes the energy of the incoming electromagnetic wave to transmit a new electromagnetic wave that contains RFID tag-specific information. The information usually contains a unique serial number. The reader receives and visualizes the information. RFIDs can be operated in different frequency bands. While the low-frequency (LF) $(30-300 \mathrm{KHz})$ and high-frequency (HF) (3-30 MHz) bands use magnetic flux coupling, the ultra-high-frequency (UHF) $(300 \mathrm{MHz}-3 \mathrm{GHz})$ band uses electromagnetic wave coupling. The communication distance of the UHF band is $>16 \mathrm{~m}$, which outperforms the LF and $\mathrm{HF}$ bands. In this work, the proposed antenna operates in the UHF band.

\section{Sensor constitutes}

The sensor we present in this work consists of an RFID chip and a half-dipole antenna. The antenna consists of eutectic liquid alloy-filled microfluidic channels in an elastic silicone substrate. The eutectic alloy we used is Galinstan. Galinstan ${ }^{35}$ is a family of room temperature liquid eutectic alloys consisting of gallium $(\mathrm{Ga})$, indium (In), and tin (Sn, Stannum in Latin). Gallium-based room temperature liquid alloy has previously been used in strain, ${ }^{16,36-38}$ pressure, ${ }^{16,36,39}$ curvature, ${ }^{19,40}$ and shear ${ }^{41}$ sensors. It can be easily injected into channels of soft structures. The resistance of a channel can be approximated by $R=\frac{\rho}{A} * l$ ( $\rho$ is resistivity of the liquid metal and $A$ and $l$ are cross-section area and length of the channel, respectively); so the channel resistance changes through deformation.

The Galinstan we made consists of $68.5 \% \mathrm{Ga}, 21.5 \% \mathrm{In}$, and $10 \% \mathrm{Sn}$; its melting temperature is $-19^{\circ} \mathrm{C}$; and its electrical conductivity is $3.46 \times 10^{6} \mathrm{~S} \times \mathrm{m}^{-1}$. ${ }^{36}$ Galinstan indicates favorable properties as channel conductor. We observed reliable liquid distributions in the antenna channels despite extreme twisting, stretching, and folding.

We used Ecoflex as the elastic silicone substrate. This silicone material has a Young's modulus of $\sim 69 \mathrm{kPa}^{42} \mathrm{We}$ chose Ecoflex to be the substrate due to its favorable properties; it is easy to synthesize, easy to handle, low cost, and biocompatible. $^{43}$ In tensile tests with the ASTM D412 system, the maximum elongation of Ecoflex 00-50 (type 00-50) is $980 \%$ (defined as the percentage change in length or $\left.\left(\left(l-l_{0}\right) / l_{0}\right) \times 100 \%\right)$.

\section{Working principles}

Figure 1a depicts a stretchable meandered half-wave dipole antenna. We embedded an RFID chip into the antenna design. Supplementary Figure S1 indicates specific antenna dimensions.

The RFID chip is bonded on aluminum pads and inserted in between two Galinstan channels. The RFID chip consumes
FIG. 1. (a) Schematic diagram of the stretchable microfluidic sensor. Galinstan is enclosed in Ecoflex substrate while an RFID chip is bonded on a piece of aluminum foil, which is inserted in the channels. Detailed and cross-section figures of the part near RFID chip are presented. Directions of $x, y$, and $z$ axes are indicated. (b) Initial state of the antenna when it is not stretched. The effective antenna length on the $x$-direction and $y$ direction is defined as $l_{x}$ and $l_{y}$, respectively. Notice: the electrical length of the entire antenna is $l_{x}+$ $10 l_{y}+l_{m}$. The nonsymmetric part, $l_{m}$, is used for fine impedance matching between the antenna and the RFID chip. (c) When the soft antenna is stretched, the effective antenna length on the $x$-direction and $y$-direction is defined as $l_{x}+$ $\Delta l_{x}$ and $l_{y}+\Delta l_{y}$, respectively. RFID, radio-frequency identification. Color images are available online.
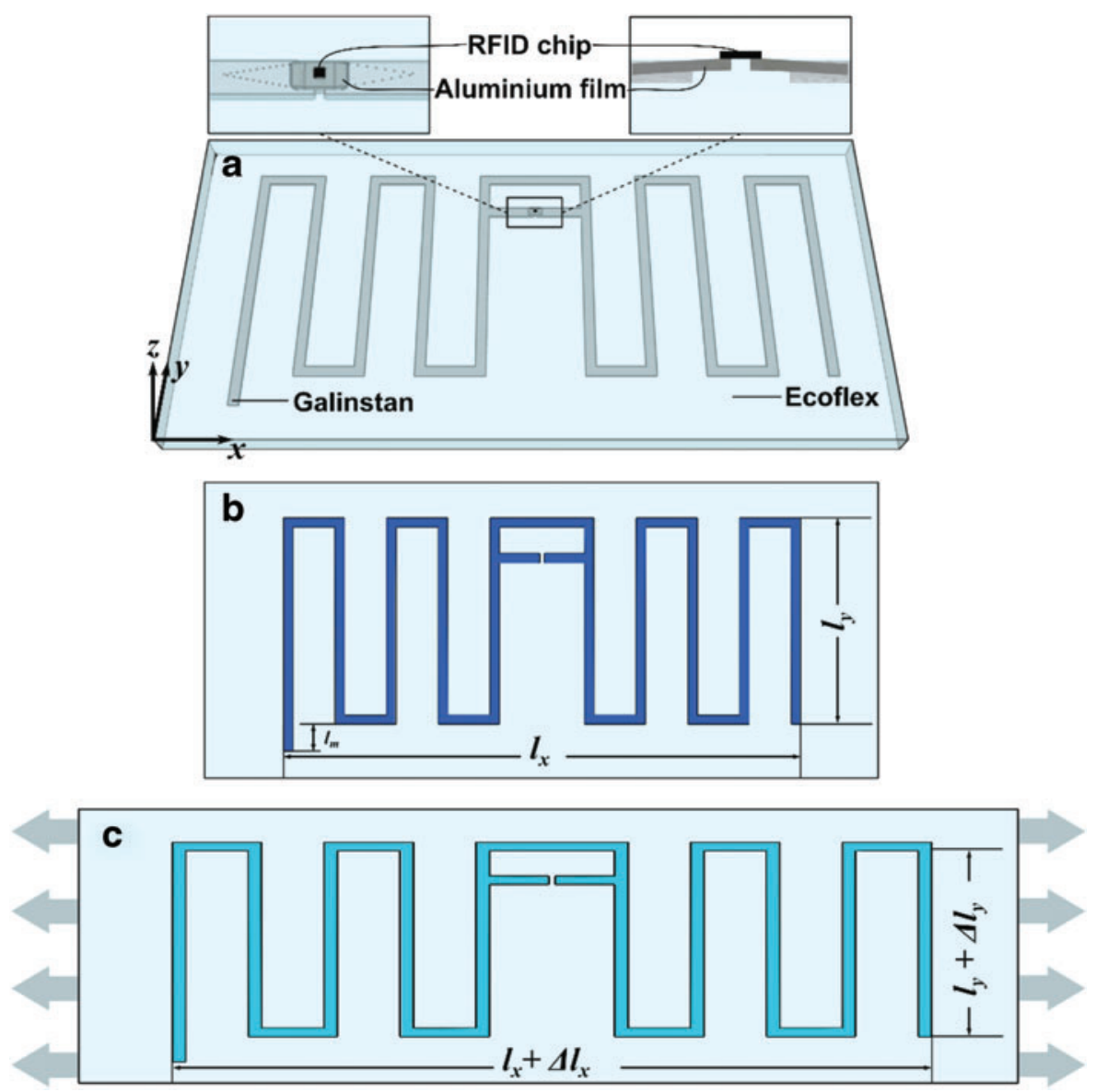
power from received signals and backscatters them without requiring an external power supply depicting a wireless passive soft sensor.

The results shown in Figure $1 \mathrm{~b}$ and $\mathrm{c}$ indicate how deformation of an antenna can change its dimensions. If the channel dimensions change, the channel resistances change and therefore the entire antenna and its parameters change. The RFID chip backscatters signals with varying amplitude and phase based on the degree of stretching.

\section{Implementation in soft robotics}

Figure 2 shows the schematic diagram of our soft RFID sensor implementation. The implementation comprises a pneumatic soft robot with four embedded soft RFID tags, one tag on each robot leg, and the electronic circuitry to transceiver data. The Controller uses the Signal Generator to generate a HF carrier signal. An Amplitude Shift Keying (ASK) module modulates the amplitude of the carrier signal to decode information. A binary "one" consists of a fixed amplitude and frequency; a binary "zero" is a zero-voltage output.

The modulated signal is passed through a power amplifier preparing the signal for transmission. The signal lies on port 1 of a circulator circuit. Port 2 of the circulator outputs the signal to an antenna, therefore broadcasting the signal. The soft RFID tags receive the signal, utilize its energy, and transmit a new signal that incorporates their unique identification information. The antenna receives the signals from the soft RFID tags and outputs them to port 3 of the circulator. The circulator ensures that outgoing and incoming signals do not interfere with one another. The incoming signals from the soft RFID tags pass a low-noise filter, an ASK demodulator, and are ultimately inserted into an analog-to-digital converter. The controller analyses the various signals (distinguishable through their identification information) with regard to their amplitude and phase.

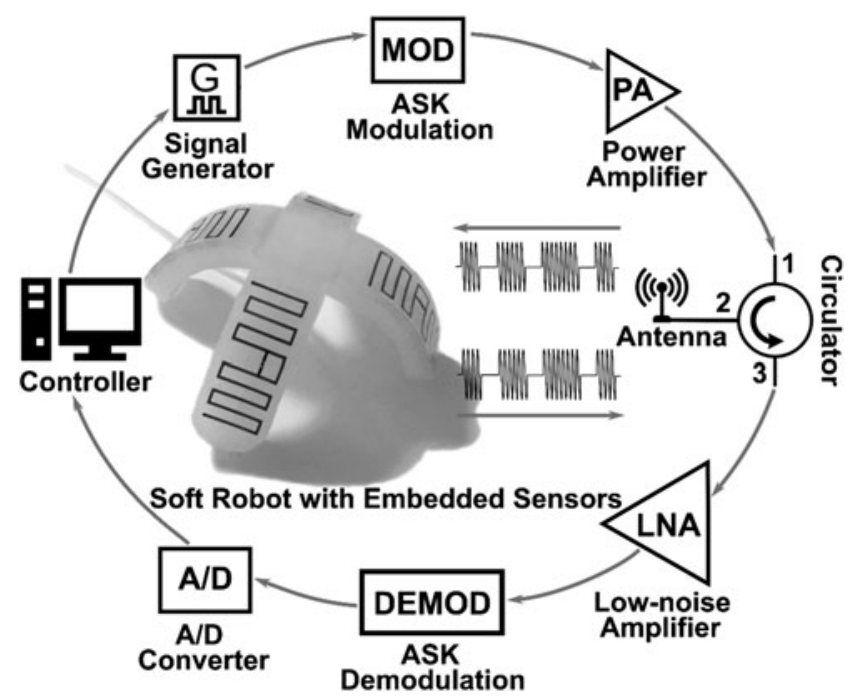

FIG. 2. The electronic system for measuring data from a pneumatic soft robot that contains four embedded microfluidic strain sensors.

\section{Antenna design}

Selection and design of the antenna. The antenna design of our soft RFID sensor is different from other antenna designs because of power and size requirements. The antenna powers the RFID chip with incoming signals; we do not require a power supply. We decided to design the soft RFID sensor as small as possible to attach the RFID tags to even small objects making our sensor more applicable. Since the soft RFID sensors can be attached in any arbitrary orientation, we ensured $360^{\circ}$ or omnidirectional readouts by using a half-wave dipole antenna design. The effective electrical length of a half-wave dipole antenna is half the wavelength of the operating frequency of the system. This antenna design is commonly used in RF systems with omnidirectional radiation patterns perpendicular to the antenna axis.

In previous work, small antenna sizes were accomplished by using microstrips. ${ }^{44}$ However, they suffered directional readout constraints and low efficiency. Our design uses a meander line antenna (MDA) to achieve long reading ranges and small antenna size. MDA is a derivative of the dipole antenna and achieves high efficiencies ${ }^{45}$ for small antenna sizes. $^{46,47}$

Stretching the soft half-wave dipole antenna as illustrated in Figure $1 \mathrm{~b}$ causes a change in the dimensions if the antenna is in both the $x$ and $y$ directions. An increase in strain in the $x$ direction decreases the thickness of the soft polymer, due to the Poisson effect, and also decreases its height by $\Delta l_{y}$. Therefore, stretching the soft RFID tag changes the electric length of the antenna; each infinitely small change in electric length shifts the resonant frequency. Now, if we send signals to RFID tags, the frequency of the carrier signal can be changed to the specific resonant frequency of the antenna's current electric length. The backscattered signals from the RFID tags will indicate phase and amplitude changes due to the resonant frequency.

Antenna matching. As mentioned in the previous section, the RFID antenna utilizes power from the incoming electromagnetic waves to supply power to the RFID chip. Impedance matching between the RFID chip and the antenna is paramount in RFID antenna design because it affects how much power the antenna is able to reflect, hence determines the antenna's operational range.

Figure 3 indicates how to match impedances. The best impedance matching for maximum power transmission is to conjugate impedance matching, which means $R_{a}=R_{c}$ while $X_{a}=-X_{c}$. The RFID chip we used (Monza R6, Impinj) possesses an impedance of $Z_{c}=(13.5-j 126) \Omega$ at $860 \mathrm{MHz}$. Therefore, we designed the transporter antenna to match the impedance of this RFID chip.

The theoretical impedance of a half-wave dipole antenna in free space is $73+j 42.5 \mathrm{Ohms},{ }^{48}$ which makes it impossible to match the impedance of the RFID chip. Therefore, we used the T-match method ${ }^{49}$ to match the impedance between transporter antenna and RFID chip. The results shown in Figure $3 \mathrm{~b}$ and $\mathrm{c}$ indicate that a half-wave dipole antenna can be changed through a centered short-circuit stub. The antenna source is connected to a small dipole of length $a \leq l$ and is placed at a close distance $b$ from the first and larger dipole. The electric current distributes along the two main radiators according to the size of their transverse sections. The impedance $Z_{a}$ at the source points is given by Equation 1: 

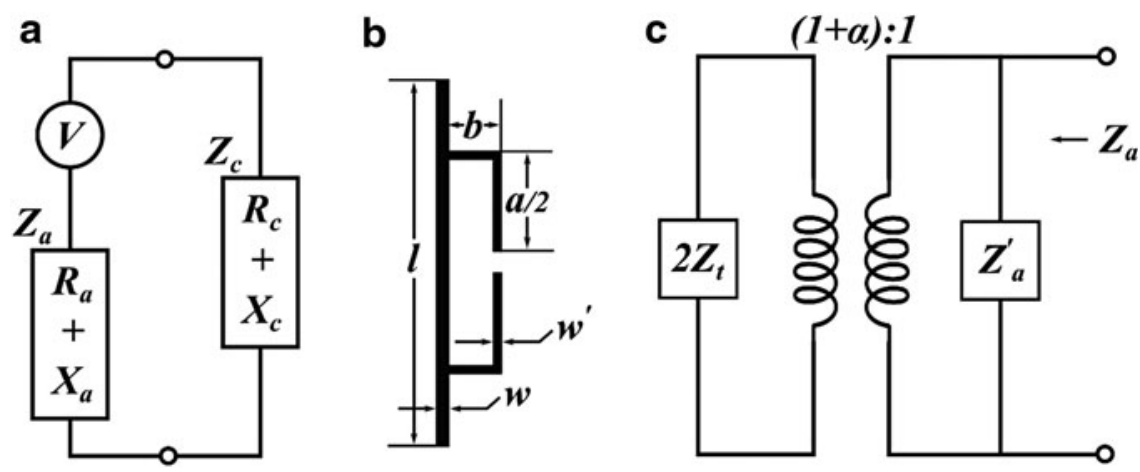

FIG. 3. (a) Equivalent circuit of the RFID chip and the transporter antenna. $Z_{a}$ is the total impedance of the transport antenna, whereas $Z_{c}$ is impedance of the RFID chip. (b) Schematic diagram of T-matching for a dipole antenna. The $l$ represents the length of the original dipole antenna. This antenna is connected at the center to a short second dipole. The close distance between matching stub and the original antenna is $b$, whereas $w$ and $w$ ' are the widths of the original dipole antenna and the mating stub, respectively. (c) Equivalent circuit at the source point of the dipole antenna after T-matching. $Z^{\prime}{ }_{a}$ is the impedance of the first dipole antenna without T-matching, whereas $Z_{t}$ is the impedance that the matching stub created. The $\alpha$ in the ratio is the current division factor between the two connectors.

$$
Z_{a}=\frac{2 Z_{t}(1+\alpha)^{2} Z_{a}^{\prime}}{2 Z_{t}+(1+\alpha)^{2} Z_{a}^{\prime}}
$$

In Equation $1, Z^{\prime}{ }_{a}$ is the original dipole impedance taken at its center in the absence of a T-match connection, whereas $Z_{t}$ is the impedance created by the matching stubs. $\alpha$ is the current division factor between the two connectors, $\alpha=\ln \left(b / r e^{\prime}\right) / \ln (b / r e)$. re and $r e$ ' are the equivalent radii of the initial dipole and the matching stub.

We performed impedance calculation with finite element method (FEM) simulations with Computer Simulation Technology (CST) Microwave Studio. FEM includes the use of mesh generation techniques for dividing a complex structure into a large number of small elements. Calculations are made for every single element, and combining the individual results can give us the conclusive results of the structure. We modified the edge of the antenna to be asymmetric, so that created $l_{m}$ in Figure $1 \mathrm{a}$, for a final fine matching step.

\section{Materials and Fabrication Methods}

Figure 4 shows the soft-lithography fabrication process of our stretchable antenna (photographs of the fabrication process are shown in Supplementary Figure S2). We designed and fabricated an antenna mould with a laser micromachining system (Protolaser U3; LPKF) and a selfadhesive vinyl film (CRAFTRKZO; $d$-c-fix ${ }^{\circledR}$ ), as shown in Figure $4 \mathrm{a}$. A piece of vinyl film was attached to an acrylic substrate ( $2 \mathrm{~mm}$ Acrylic Cast; AMARI). The channel profiles were cut with the LPKF laser system. We peeled the vinyl residuals (negative) from the substrate and laid a $2 \mathrm{~mm}$-thick acrylic frame along the mould edges as indicated in Figure $4 \mathrm{~b}$.

We synthesized Ecoflex 00-50 (Reynolds Advanced Materials; $1: 1$ ratio), degassed it in a vacuum chamber, and poured the soft polymer into the mould as illustrated in Figure $4 \mathrm{c}$. We put the mould into a convection oven at $65^{\circ} \mathrm{C}$ for $20 \mathrm{~min}$ and then removed the soft structure from the mould and flipped it over. A $150 \mu$ m-thin Ecoflex 00-50 layer was fabricated with spin coater to seal the unfolded channels, as shown in Figure $4 d$.
We made small incisions in the center of the thin Ecoflex layer to embed the RFID chip; the RFID chip (Monza R6; Impinj) was bonded on an Aluminum film with a bonding machine (FB-300 manual RFID wrapping machine) and inserted in the channels. During the bonding process, the chip was aligned to the substrate with a vacuum sucker, microscopes, and cameras in the bonding machine, an adhesive material was dispensed between the chip and the aluminum film, then the chip was firmly bonded on the aluminum film with hot-pressing for $10 \mathrm{~s}$.

We inserted two injection needles into the soft structure, as illustrated in Figure 4e. One needle was used to inject the Galinstan into the channel, and the other needle was used to release air pressure. The Galinstan made contact with the aluminum pads of the RFID chip without leaking to the surface. At the end, we applied a thin film of uncured Ecoflex on top of the device to seal the open holes created by chip insert and liquid metal injection.

\section{Experimental Design}

The permittivity of Ecoflex 00-50 and the resistivity of Galinstan are parameters that are required to simulate $S_{11}$; $S_{11}$ is the input reflection coefficient or input return loss. $S_{11}$ indicates the radiation efficiency of the antenna and reaches its maximum value at resonant frequency. We used an Agilent $85070 \mathrm{E}$ dielectric probe kit to measure the permittivity variations of Ecoflex 00-50 in a frequency range from $200 \mathrm{MHz}$ to $1.2 \mathrm{GHz}$, as shown in Figure $5 \mathrm{~b}$. We simulated changes in resonant frequency and radiation efficiency under strain variations between $0 \%$ and $50 \%$ and a step size of $5 \%$.

We used a Voyantic Tagformance system to measure the characteristics of our antenna design; namely the frequency modulation of our soft RFID sensor due to strain variations, as indicated in Figure 5a and c. This setup allowed us to determine the resonant frequency, the theoretical RFID reading range, and the phase changes of the backscattered signals for every applied strain in the range from $0 \%$ to $50 \%$ with a step size of $5 \%$. 

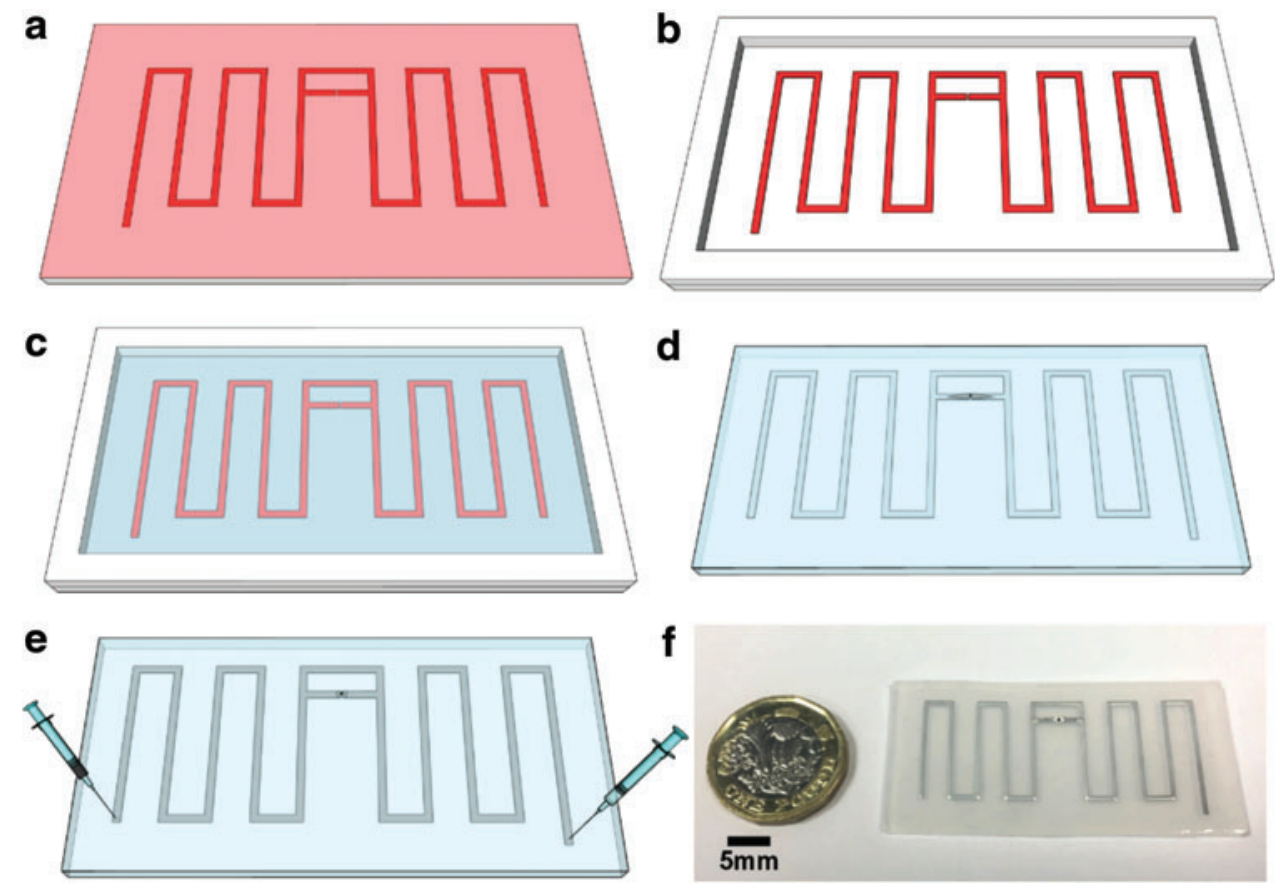

FIG. 4. Fabrication process and prototype of the stretchable microfluidic sensor (RFID tag antenna). (a) Cut the sensor profile on a piece of self-adhesive vinyl attached on an acrylic substrate. Notice: the red and pink parts are a same piece of vinyl. Two different colors are used here to show the laser-cut profile clearer. (b) The unwanted part of vinyl was peel off, a 2 mmthick acrylic frame was stuck on the substrate to form a soft-lithography mold. (c) Uncured Ecoflex with a controlled height of $2 \mathrm{~mm}$ was poured into the soft-lithography mould. (d) The casted piece of Ecoflex was sealed with another $0.15 \mathrm{~mm}$ Ecoflex film, and the RFID chip bonded on aluminum film was inserted into the channels. (e) Two needles were used to inject Galinstan into the channel. (f) Photograph of the final prototype. Color images are available online.
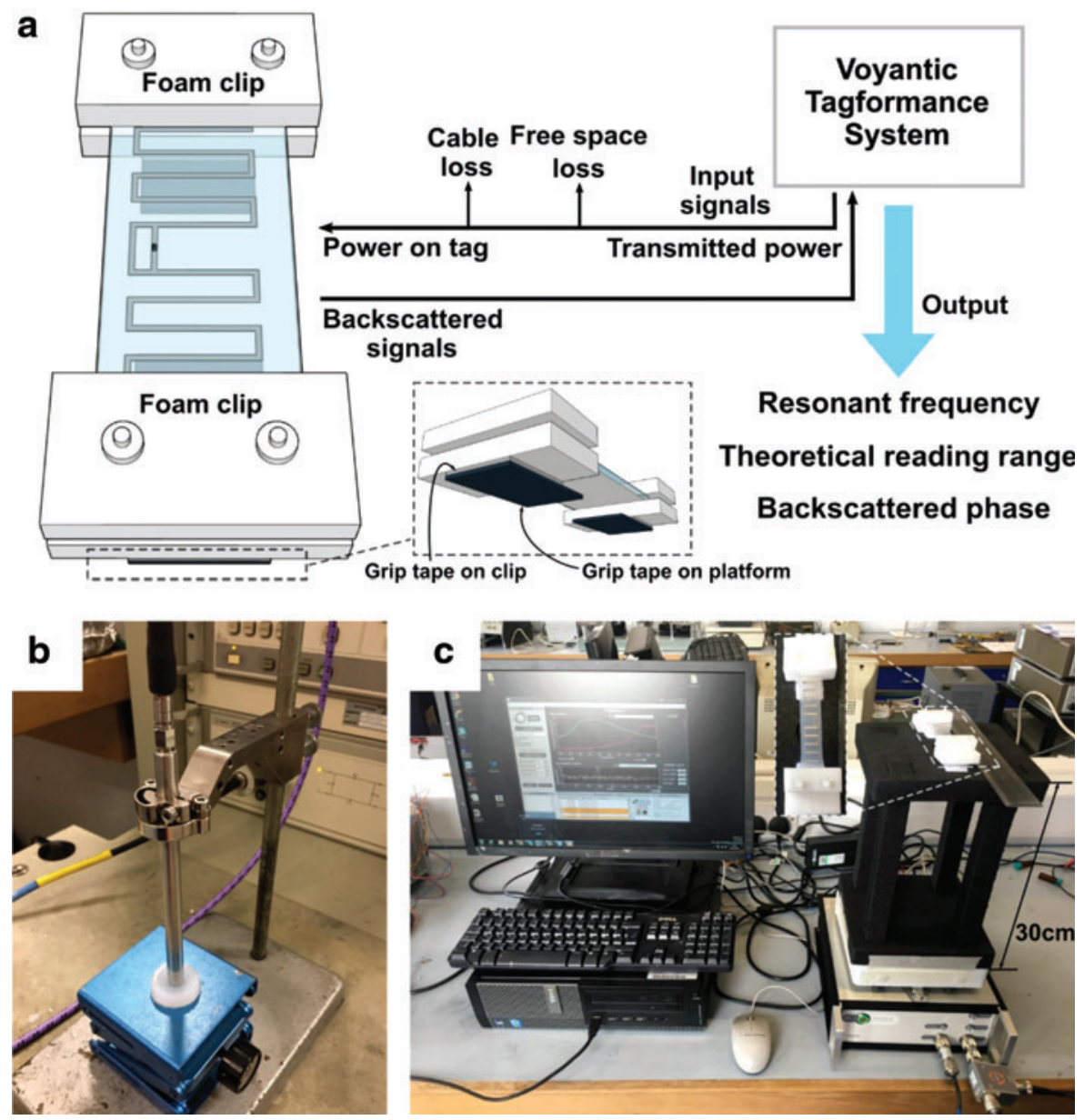

FIG. 5. (a) We used the Voyantic Tagformance system to measure characteristics of the proposed antenna. This measurement system sends input signals to the tag antenna with transmitted power. The power received on tag is the transmitted power minus cable loss and free space loss. RFID chip in the tag antenna receives the input signals, absorbs energy in the signals, and then sends backscattered signals back to the measurement system. According to the results of the measurement system, we can know the resonant frequency and the theoretical reading range of the tag antenna, as well as the phase of the backscattered signals. We affixed the two edges of the tag antenna with clips made of rigid foam. To stretch the tag antenna and hold its position for measurement, we stuck grip tape on the surface of the foam clips and on the measurement platform. (b) Experimental setup for measurement of the permittivity of Ecoflex 00-50 with the Agilent $85070 \mathrm{E}$ dielectric probe kit system. (c) Photograph of the experimental setup of the soft antenna and the Voyantic Tagformance system. Color images are available online. 
FIG. 6. Radiation pattern measurement in the (a) $\mathrm{H}$ plane and the (b) E-plane in an anechoic room with Diamond Engineering Automated measurement system and an automatic turntable. In the measurement, the reader antenna holds its position while the strain sensor (transporter antenna) turns with the turntable. Color images are available online.
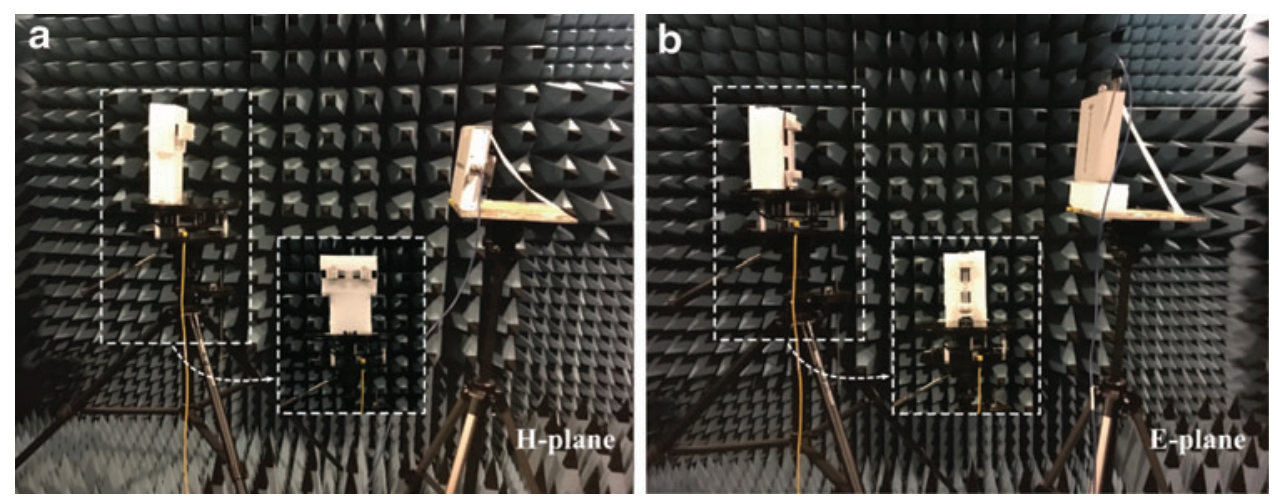

We repeated each measurement seven times to indicate the soft polymer's reversibility. We fabricated three sensor samples to enable us to evaluate the repeatability of our fabrication process. We did a cyclic stretching test to prove the reliability of the proposed sensor when it is stretched to $50 \%$ for 240 times. It is worth mentioning that we calculated the strain value in all the experiments with $\left(\left(\frac{\Delta l_{x}}{l_{x}}\right) \times 100 \%\right)$, $l_{x}$ and $\Delta l_{x}$ are shown in Figure 1. The length we used in calculation is the effective antenna length in the $x$-direction, not the distance between the foam clips. We also tested the soft RFID sensors when we applied twists of $0^{\circ}, 45^{\circ}$, and $90^{\circ}$.

We used a diamond engineering automated measurement system and an automatic turning table in an anechoic room to measure the radiation patterns of our soft RFID sensor in the H- and E-planes (Fig. 6). An anechoic room absorbs reflections from acoustic and electromagnetic waves. We analyzed the directivity of the antenna (in the $\mathrm{H}$ - and E-planes) when subjected to strains of $0 \%, 25 \%$, and $50 \%$.

\section{Results}

\section{Permittivity of Ecoflex 00-50}

According to the results in Figure 7, the relative permittivity $\left(\varepsilon_{r}\right)$ of Ecoflex 00-50 is nearly stable at 3 in the range of $500 \mathrm{MHz}-1.2 \mathrm{GHz}$, as shown in Figure 7. The operating frequency range of our sensor is $800-860 \mathrm{MHz}$, which is covered in this stable range. Thus, we can take $\varepsilon_{r}$ of Ecoflex as a constant in calculation and simulation.

\section{Simulation of antenna efficiency}

When our soft RFID sensor is stretched, its antenna parameters such as resonant frequency and radiation efficiency change. Below, we analyze, analytically, the antenna parameters due to strain variations and we apply an ideal folded half-wave dipole antenna model.

The resonant frequency of a half-wave dipole antenna ${ }^{50}$ is given by

$$
f_{r}=\frac{c}{2 l_{e} \sqrt{\varepsilon_{e}}} \times r
$$

where $l_{e}$ is the effective electrical length of the antenna; $f_{r}$ is the resonant frequency; $\varepsilon_{e}$ is the effective permittivity of the antenna substrate; and $r$ is the correction factor of a folded half-wave dipole antenna. We can estimate the effective relative permittivity through the study of Jackson et al. ${ }^{51}$ :

$$
\varepsilon_{e}=1+\left(\frac{\varepsilon_{r}-1}{2}\right) \frac{K_{1}}{K_{2}},
$$

where $\varepsilon_{r}$ is the relative permittivity of Ecoflex, which is considered a constant here; $K_{1}=\frac{K\left(k_{1}\right)}{K^{\prime}\left(k_{1}\right)}$ and $K_{2}=\frac{K\left(k_{2}\right)}{K^{\prime}\left(k_{2}\right)}$ while $K(k)$ is a complete elliptic function of the first kind and $K\left(k^{\prime}\right)$ is its complimentary function.

In the $\mathrm{K}$ function, $k_{1}=\frac{l_{e}}{l_{e}+2 s}$ and $k_{2}=e^{-\frac{\pi s}{2 h}}$, whereas $l_{e}$ is effective electrical length of the antenna, $s$ is the gap at the feed point, and $h$ is height of substrate of the antenna. According to Equations 2 and 3, resonant frequency of a halfwave dipole antenna will change when its effective electrical length or its substrate height changes.

Equation 2 shows the resonant frequency of a half-wave dipole antenna changes with its effective electrical length, whereas Equation 3 indicates that the resonant frequency also changes with the substrate height of the antenna.

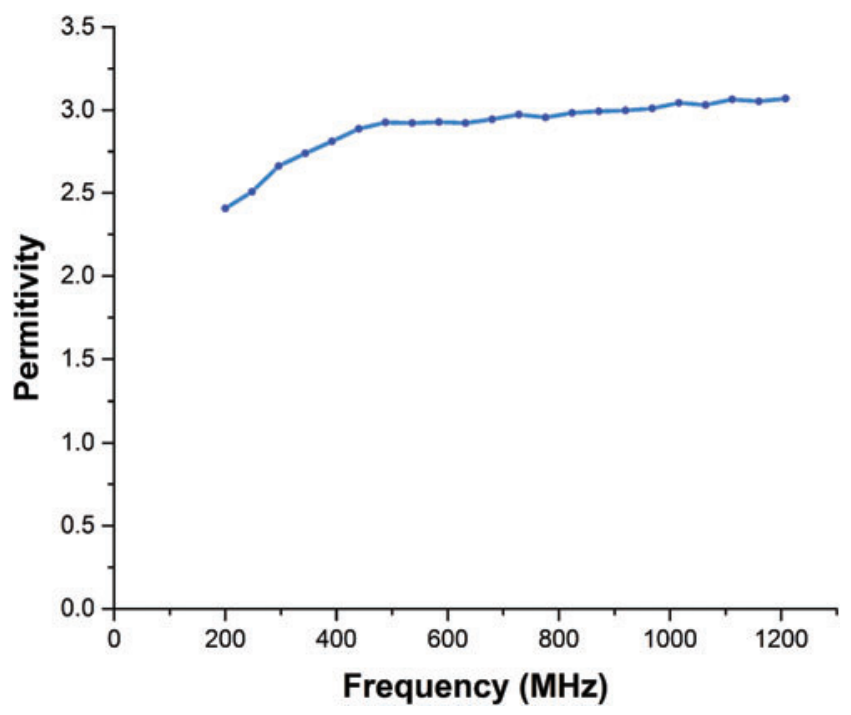

FIG. 7. Permittivity of Ecoflex type 00-50 in the frequency range of $200 \mathrm{MHz}-1.2 \mathrm{GHz}$. Measured with Agilent $85070 \mathrm{E}$ dielectric probe kit system. Color images are available online. 
Rigid antennas possess a fixed effective electrical length, whereas our soft antenna's effective electrical length changes due to strain. Due to the Poisson effect, our antenna physically changes according to

$$
\frac{\Delta l_{y}}{l_{y}}=1-\left(1+\frac{\Delta l_{x}}{l_{x}}\right)^{-\nu}
$$

where $l_{x}$ is the antenna length; $\Delta l_{x}$ describes a change in antenna length; $l_{y}$ is the antenna width; $\Delta l_{y}$ describes a change in antenna width; and the initial (not stretched) electrical length of our antenna is $l_{x}+10 l_{y}+l_{m}$. Since $l_{m} \gg l_{x}+10 l_{y}$, we neglect the effect of $l_{m}$ here. Figure 1 complements Equation 4.

We insert Equations 3 and 4 in Equation 2 and receive a new formulation for the resonant frequency for our soft antenna

$$
f_{r}=\frac{c}{2\left(l_{x}\left(1+\frac{\Delta l_{x}}{l_{x}}\right)+10 l_{y}\left(2-\left(1+\frac{\Delta l_{x}}{l_{x}}\right)^{-\nu}\right)\right) \times \sqrt{1+\left(\frac{\varepsilon_{r}-1}{2}\right) \frac{K_{1}}{K_{2}}}} \times k,
$$

where $\nu$ is the Poisson ratio of Ecoflex type 00-50.

We performed an electromagnetic finite element simulation using CST to determine $S_{11}$, the input return loss, under a variety of strains. $S_{11}$ 's maximum indicates the antenna's resonant frequency. Figure 8 indicates the simulation results; the soft antenna has been strained from $0 \%$ to $50 \%$ with a step size of 5\%; the $S_{11}$ peak values are all below $-20 \mathrm{~dB}$ at resonance frequency for each strain condition indicating that 99\% of the power from the input signal is scattered back from the soft RFID sensor. We successfully matched antenna to RFID chip resistance.

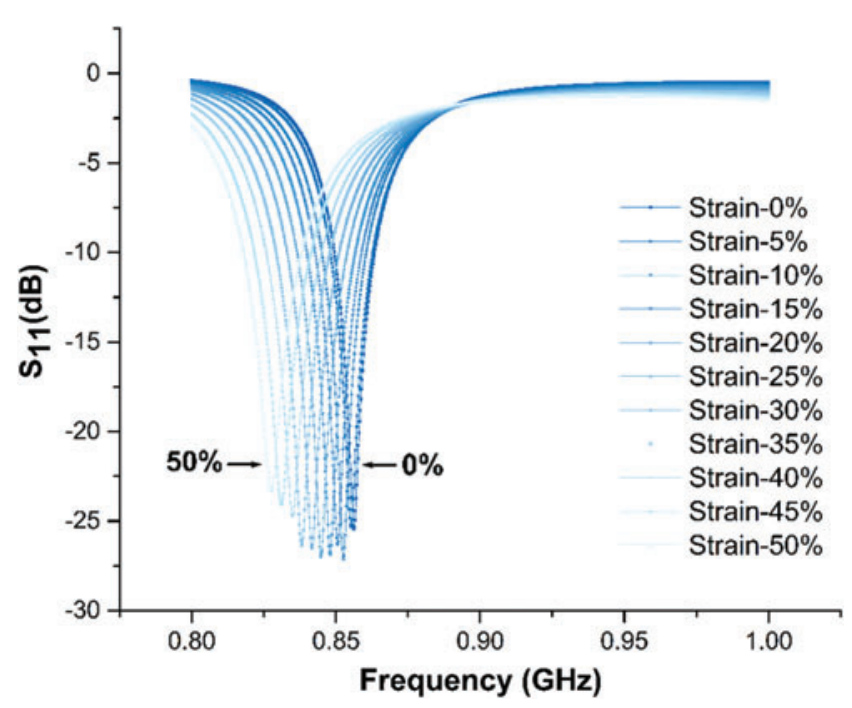

FIG. 8. Finite elements simulation results. Under $0 \%$ strain condition, simulated resonant frequency of the antenna is $856 \mathrm{MHz}$. The simulated resonant frequency continuously goes down to $827 \mathrm{MHz}$ when the antenna is under $50 \%$ strain. Color images are available online.

\section{Resonant frequency upon strain}

The Voyantic Tagformance measurement system controls the power of the signal that we transmit to the soft RFID sensor. First, we set the initial signal power to $25 \mathrm{~dB}$. Second, we gradually decreased the signal power until we could not detect the backscattered signal from the soft RFID sensor anymore. Power of the input signal was recorded as Transmitted Power $\left(P_{t}\right)$. We repeated this process for each sampling frequency in the frequency band of $800-880 \mathrm{MHz}$, with a step of $1 \mathrm{MHz}$.

As indicated in Figure 5a, the power received by the soft RFID tag antenna $\left(P_{t a g}\right)$ can be calculated as

$$
P_{t a g}=P_{t}-L_{c}+G_{t}-F S P L,
$$

where $L_{c}$ are cable losses; $G_{t}$ is the Gain of the Voyantic Tagformance's transmitter antenna; and $F S P L$ are losses due to free space transmissions. $L_{c}, G_{t}, F S P L$ can be determined by calibrating the Voyantic Tagformance.

Figure 9a shows the minimum transmitted power $\left(P_{t}\right)$ for each sample frequency to result in a detectable backscattered signal from the soft RFID sensor. Figure 9b indicates the power received on tag antenna (power on tag, $P_{\text {tag }}$ ) corresponding to the minimum $P_{t}$ for each sample frequency.

The theoretical measurement reading range of the soft RFID sensor is frequency dependent and can be calculated by

$$
R_{r}=\sqrt{\frac{P_{\max , E I R P}}{P_{\text {tag }}}} \times \frac{c}{4 \pi f},
$$

where $P_{\max , \text { EIRP }}$ is the maximum signal power of the Voyantic Tagformance (default: $3.28 \mathrm{~W}$ ). $P_{\text {tag }}$ in this formula is related to the minimum transmitted power. Figure $9 \mathrm{c}$ depicts the reading ranges calculated with Equation 7 for the various frequencies; our soft RFID sensor has a reading range of $\sim 7.5 \mathrm{~m}$, whereas this range increases if the soft sensor is under strain. The reading range of the sensor increases to $10 \mathrm{~m}$ under a strain of $50 \%$. The resonant frequency decreases from $862 \mathrm{MHz}$ in idle state (no strain) to around $802 \mathrm{MHz}$ under 50\% strain. Figure 9d illustrates the backscattered signals from the soft RFID sensor under different strain conditions.

\section{Repeatability and reliability test}

We tested the reliability of our soft RFID sensor by performing seven strain experiments. We measured the resonant frequency under $0 \%-50 \%$ strain, with a step size of 5\%. Figure 10a shows the average value and standard deviation of the resonant frequency for each strain. The resonant frequency dropped $\sim 61 \mathrm{MHz}$ (from 861 to $800 \mathrm{MHz}$ ) from $0 \%$ to $50 \%$ strain, whereas the maximum standard deviation did not exceed $2.6 \mathrm{MHz}$.

We performed a similar experiment in Figure 10c; once we reached $50 \%$ strain, we left the device stretched for $24 \mathrm{~h}$ in the laboratory before we gradually released the device to $0 \%$ strain. We also involved the simulation results of resonant frequency upon strain in Figure 10a as a comparison with the measurement results. In the simulation, the resonant 

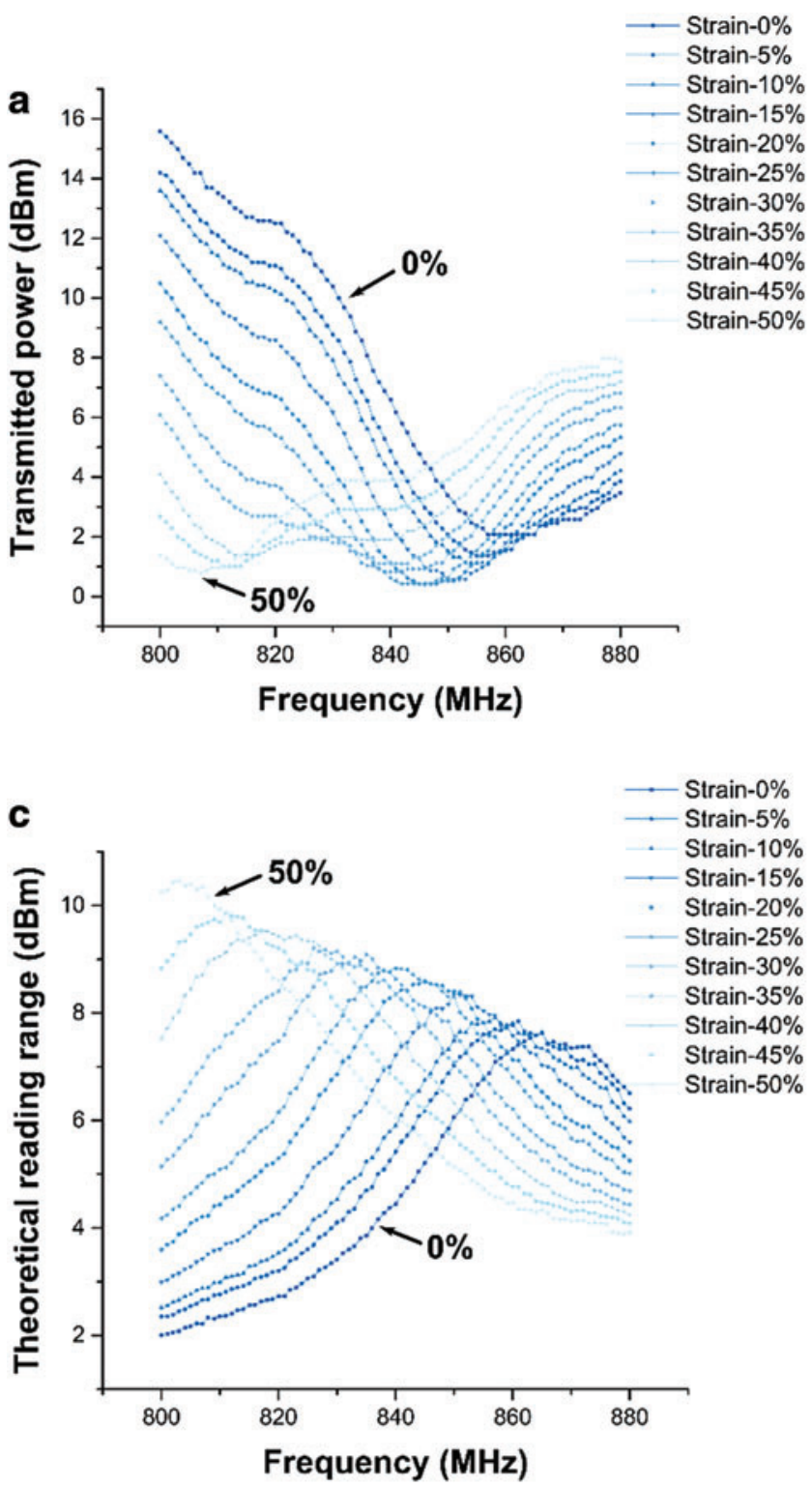
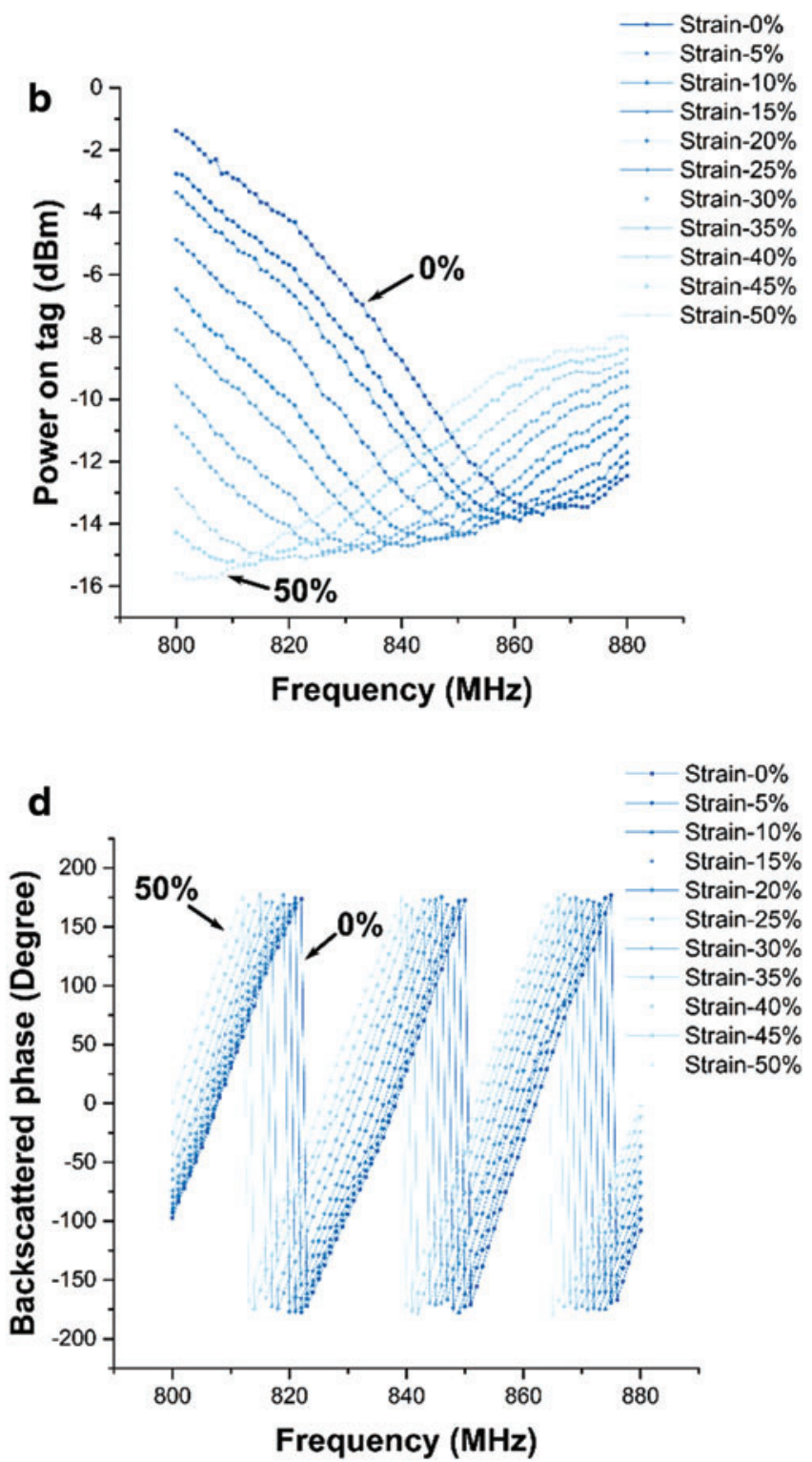

FIG. 9. (a) Transmitted power. The minimum transmitted power recorded for the sensor under $0 \%-50 \%$ strain, with a step of 5\%. (b) The power received on tag, which was calculated based on the transmitted power and cable and free space loss. (c) Theoretical reading range forward, which was calculated based on the power received on tag. (d) Backscattered phase in the frequency band of $800-880 \mathrm{MHz}$ (with a step of $1 \mathrm{MHz}$ ) when the sensor was under $0 \%-50 \%$ strain, with a step of $5 \%$. Color images are available online.

frequency changed $29 \mathrm{MHz}$ in total (from 856 to $827 \mathrm{MHz}$ ) from $0 \%$ to $50 \%$ strain, which is smaller than the measurement results.

We fabricated another three prototypes of the same sensor design and again performed strain-resonant-frequency tests. Average value and standard deviation of the resonant frequency for each prototype are shown in Figure 10b. Figure 10b shows similar results than Figure 10a. Figure 10d depicts a cyclic s stretching test to prove the reliability of the proposed sensor when it is stretched 240 times.

As shown in the results, the resonant frequency of the sensor remains stable in the test (only dropped $2 \mathrm{MHz}$ between 80 and 100 of iteration). We also measured resonant frequency response of a soft RFID sensor due to twist rather than strain and put the results in Supplementary Data. As shown in Supplementary Figures S3 and S4, three different twist angles were tested. According to the results, the electrical characteristics of our soft RFID sensor at frequency of operation are highly sensitive to mechanical strain while having no response to twisting.

\section{Radiation pattern}

We measured the theoretical reading range from our soft RFID sensor in the $\mathrm{H}$ - and E-planes with an antenna measurement system consisting of antenna measurement studio 5.5 and Diamond Engineering. The left subfigure of Figure 11 shows that omnidirectional radiation patterns were measured 

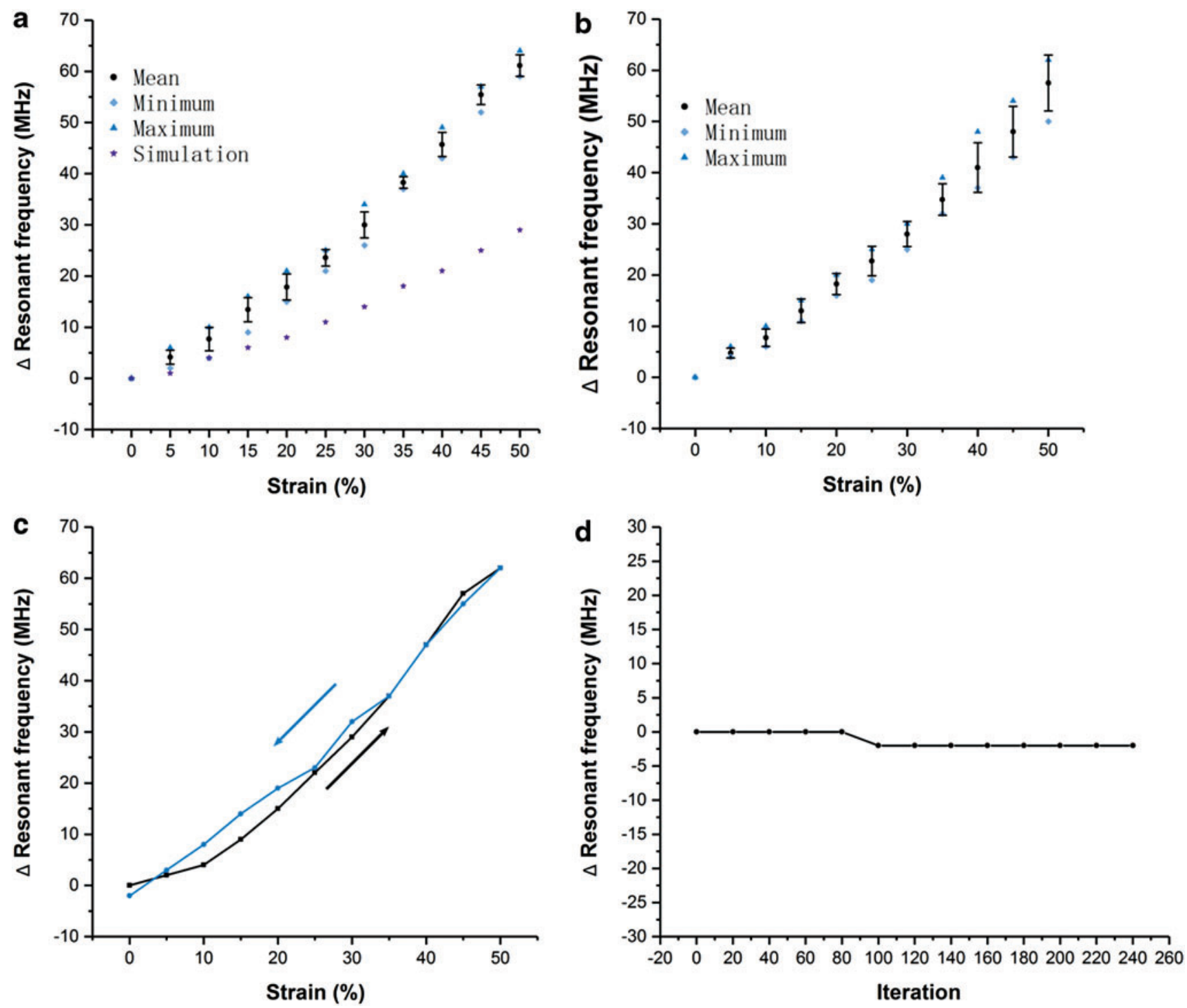

FIG. 10. (a) Resonant frequency change upon different strain conditions. In the experiment, we did seven tests on the same prototype. Error bars represent standard deviation. (b) Resonant frequency change upon different strain conditions. In the experiment, we did one test on three different prototypes with the same design and same fabrication method. Error bars represent standard deviation. (c) Hysteresis test. A prototype was tested from 0\% to 50\% of strain, leave for $24 \mathrm{~h}$, then from $50 \%$ back to $0 \%$. (d) Cyclic stretching test. The sample was stretched to $50 \%$ of strain and then back to $0 \%$ for 240 times. Resonant frequency of the sample was measured each 20 times of stretching. Color images are available online.

in the H-plane. The right subfigure of Figure 11 indicates that the antenna is constrained in directions that are parallel to the sensor's $x$-axis $\left(60^{\circ}+n \pi<\theta<120^{\circ}+n \pi ; n\right.$ is an arbitrary integer). This constraint might have to be considered in certain applications. The different strain conditions have an impact on our soft RFID sensor's reading range radius; if strain is applied, the reading range radius increases.

\section{Discussion}

The results show that our soft RFID sensor changes its resonant frequency and the phase of its backscattered signal as function of strain.
Our measurements correlate with our simulations, the initial resonant frequency of all our sensor prototypes lies in the region of $860 \mathrm{MHz}$. In our simulation, the resonant frequency did not decrease as much under strain as in our experiments. This mismatch could be caused by the simplified model that we used for the Poisson effect or a potentially emerging resistance between the Galinstan channels and the RFID chip that might occur under strain.

We matched the resistances of the RFID chip and the antenna under $0 \%$ strain. Once we apply strain, the resistance of the antenna changes, hence the resistances of the RFID chip and antenna do not perfectly match anymore. Therefore, we expected a decrease in the measurement reading range 

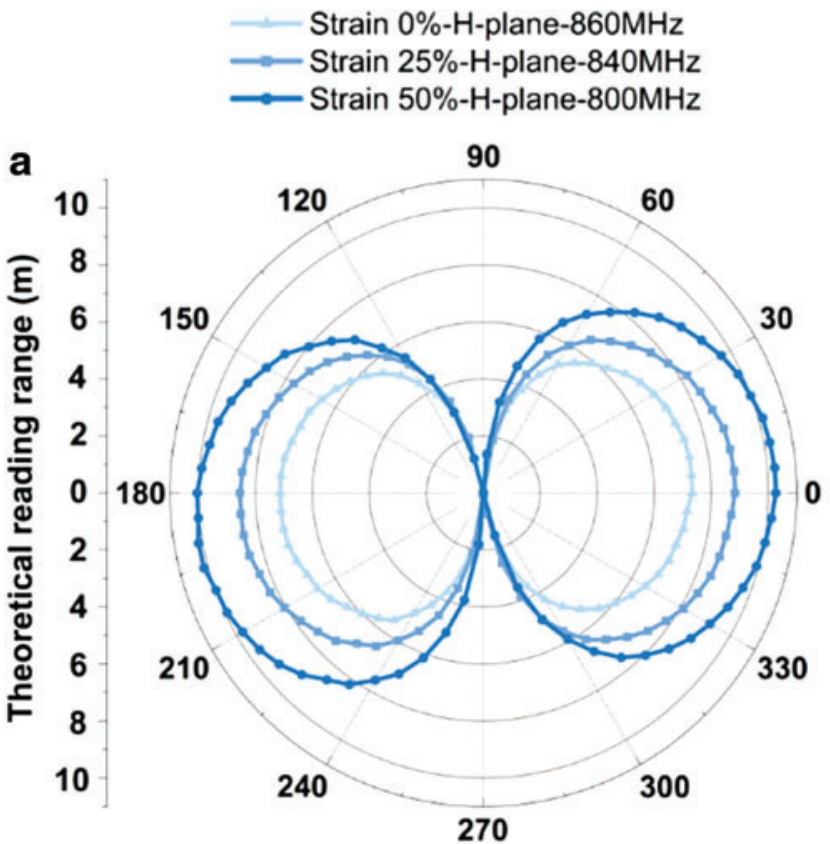

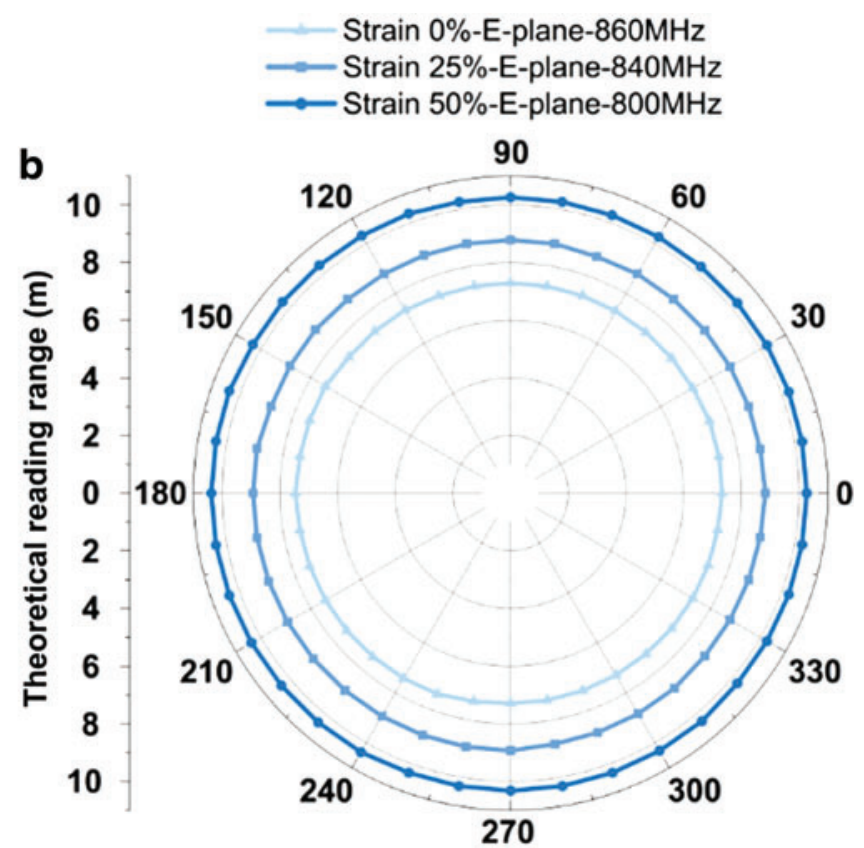

FIG. 11. Theoretical reading range of the antenna in the H-plane (a) and the E-plane (b) with $0 \%, 25 \%$, and $50 \%$ length stretching in the $x$-direction. The data were recorded for every $5^{\circ}$ rotation in the H-plane and every $10^{\circ}$ rotation in the Eplane. Color images are available online.

with an increase in strain. However, the measurement reading range increased with an increase of strain.

One explanation could be that a folded dipole antenna consists of conductors with opposite current flows; the opposing magnetic fields that are evoked through the currents might cancel each other out. Now, if we stretch the soft antenna, the conductors are being further separated and the magnetic fields might be too far away to interfere with one another. This may enhance the radiation efficiency of the antenna. An increase of the radiation efficiency might outperform an imperfectly matched antenna-RFID chip.

In this study, we investigated the resonant frequency response of stretchable soft RFID sensors due to strain. Future work might focus on the design of soft RFID sensors based on phase shift differences that occur in backscattered signals. We also want to increase the initial resonant frequency of our soft RFID sensors to increase the strain range. RFIDs usually operate in the range of $800 \mathrm{MHz}-1 \mathrm{GHz}$, whereas, currently, our system operates in the $800-860 \mathrm{MHz}$ range.

\section{Conclusion}

In this work, we designed and fabricated a stretchable strain sensor (soft RFID sensor) and tested a variety of its characteristics. The soft RFID sensor is a deformable folded half-wave dipole antenna. Its resonant frequency and backscattered signal phase changes under strain. We showed that our soft sensor is stable, reliable, and manufacturable. Our sensor consists of Ecoflex 00-50 and Galinstan, materials that are stretchable and withstand permanent deformation. The soft RFID sensor can be easily applied to for applications including wearable strain sensors or as part of soft robotic systems, as demonstrated in this study. The sensor does not require a power supply, indicates long measurement reading ranges $(>7.5 \mathrm{~m})$, and shows omnidirectivity.

\section{Acknowledgments}

The authors acknowledge funding from the Engineering and Physical Sciences Research Council: Centre for Doctoral Training in Intelligent Sensing and Measurement EP/ L016753/1, the Robotarium Capital Equipment and Robotics CDT Capital Equipment EP/L016834/1 and the UK Robotics and Artificial Intelligence Hub for Offshore Energy Asset Integrity Management EP/R026173/1.

\section{Author Disclosure Statement}

No competing financial interests exist.

\section{References}

1. Yeo WH, Kim YS, Lee J, et al. Multifunctional epidermal electronics printed directly onto the skin. Adv Mater 2013; 25:2773-2778.

2. Shademan A, Decker RS, Opfermann JD, et al. Supervised autonomous robotic soft tissue surgery. Sci Transl Med 2016;8:337ra64.

3. Polygerinos $\mathrm{P}$, Wang Z, Galloway $\mathrm{KC}$, et al. Soft robotic glove for combined assistance and at-home rehabilitation. Rob Auton Syst 2015;73:135-143.

4. Alspach A, Kim J, Yamane K. Design of a soft upper body robot for physical human-robot interaction. In: 2015 IEEERAS 15th International Conference on Humanoid Robots (Humanoids), Seoul, South Korea, November 3-5, 2015, pp. 290-296.

5. Bahramzadeh Y, Shahinpoor M. A review of ionic polymeric soft actuators and sensors. Soft Robot 2014;1: $38-52$. 
6. Wei W, Guo S. A novel PDMS diaphragm micropump based on ICPF actuator. In: 2010 IEEE International Conference on Robotics and Biomimetics, ROBIO 2010, Tianjin, China, December 14-18, 2010, pp. 1577-1583.

7. Cho MS, Seo HJ, Nam JD, et al. An electroactive conducting polymer actuator based on NBR/RTIL solid polymer electrolyte. Smart Mater Struct 2007;16:S237.

8. Shepherd RF, Ilievski F, Choi W, et al. Multigait soft robot. Proc Natl Acad Sci USA 2011;108:20400-20403.

9. Wei T, Stokes A, Webb B. A soft pneumatic maggot robot. In: Lecture Notes in Computer Science (Including Subseries Lecture Notes in Artificial Intelligence and Lecture Notes in Bioinformatics), Volume 9793, Lepora N, Mura A, Mangan M, et al. (Eds). Springer, Cham, 2016, pp. 375-386.

10. Suzumori K, Endo S, Kanda T, et al. A bending pneumatic rubber actuator realizing soft-bodied manta swimming robot. In: Proceedings-IEEE International Conference on Robotics and Automation, Roma, Italy, April 10-14, 2007, pp. 4975-4980.

11. Ilievski F, Mazzeo AD, Shepherd RF, et al. Soft robotics for chemists. Angew Chem Int Ed Engl 2011;50:18901895.

12. Cianchetti M, Mattoli V, Mazzolai B, et al. A new design methodology of electrostrictive actuators for bio-inspired robotics. Sens Actuators B Chem 2009;142:288-297.

13. Pelrine R. Dielectric elastomer artificial muscle actuators: toward biomimetic motion. Proc SPIE 2002;4695:126-137.

14. Madden JDW, Vandesteeg NAAP, Madden JDW, et al. Artificial muscle technology: physical principles and Naval prospects. IEEE J Ocean Eng 2004;29:706-728.

15. Kim D-H, Ghaffari R, Lu N, et al. Flexible and stretchable electronics for biointegrated devices. Annu Rev Biomed Eng 2012;14:113-128.

16. Park Y-L, Chen B, Wood RJ. Design and fabrication of soft artificial skin using embedded microchannels and liquid conductors. IEEE Sens J 2012;12:2711-2718.

17. Tabatabai A, Fassler A, Usiak C, et al. Liquid-phase galliumindium alloy electronics with microcontact printing. Langmuir Acs J Surfaces Colloids 2013;29:6194-6200.

18. Park YL, Chen B, Majidi C, et al. Active modular elastomer sleeve for soft wearable assistance robots. In: Proceedings of the IEEE/RSJ International Conference on Intelligent Robots and Systems (IROS'12), Vilamoura, Portugal, October 2012, pp. 1595-1602.

19. Majidi C, Kramer R, Wood RJ. A non-differential elastomer curvature sensor for softer-than-skin electronics. Smart Mater Struct 2011;20:105017.

20. Teng L, Jeronimo K, Wei $\mathrm{T}$, et al. Integrating soft sensor systems using conductive thread. J Micromech Microeng 2018;28:054001.

21. Cheng S, Rydberg A, Hjort K, et al. Liquid metal stretchable unbalanced loop antenna. Appl Phys Lett 2009;94: 144103.

22. Cheng $\mathrm{S}$, Wu Z, Hallbjörner $\mathrm{P}$, et al. Foldable and stretchable liquid metal planar inverted cone antenna. IEEE Trans Antennas Propag 2009;57:3765-3771.

23. Kubo M, Li X, Kim C, et al. Stretchable microfluidic radiofrequency antennas. Adv Mater 2010;22:2749-2752.

24. So JH, Thelen J, Qusba A, et al. Reversibly deformable and mechanically tunable fluidic antennas. Adv Funct Mater 2009;19:3632-3637.

25. Huang Y, Wang Y, Xiao L, et al. Microfluidic serpentine antennas with designed mechanical tunability. Lab Chip 2014;14:4205-4212.
26. Kim J, Banks A, Cheng H, et al. Epidermal electronics with advanced capabilities in near-field communication. Small 2015;11:906-912.

27. Cheng S, Wu Z. A microfluidic, reversibly stretchable, large-area wireless strain sensor. Adv Funct Mater 2011;21: 2282-2290.

28. Tolley MT, Shepherd RF, Mosadegh B, et al. A Resilient, Untethered Soft Robot. Soft Robot 2014;1:213-223.

29. Marchese AD, Katzschmann RK, Rus D. A recipe for soft fluidic elastomer robots. Soft Robot 2015;2:7-25.

30. Bartlett NW, Tolley MT, Overvelde JTB, et al. A 3Dprinted, functionally graded soft robot powered by combustion. Science (80-) 2015;349:161-165.

31. Wehner M, Truby RL, Fitzgerald DJ, et al. An integrated design and fabrication strategy for entirely soft, autonomous robots. Nature 2016;536:451-455.

32. Liu R, Huskic G, Zell A. Dynamic objects tracking with a mobile robot using passive UHF RFID tags. In: 2014 IEEE/ RSJ International Conference on Intelligent Robots and Systems, Chicago, IL, September 14-18, 2014, pp. 42474252.

33. Sanpechuda T, Kovavisaruch L. A review of RFID localization: applications and techniques. In: 5th International Conference on Electrical Engineering/Electronics, Computer, Telecommunications and Information Technology, ECTI-CON 2008, Vol 2, Krabi, Thailand, May 14-17, 2008, pp. 769-772.

34. Choi B-S, Lee J-W, Lee J-J. Distributed sensor network based on RFID system for localization of multiple mobile agents. Wirel Sens Netw 2011;3:1-9.

35. Liu T, Sen P, Kim CJ. Characterization of nontoxic liquidmetal alloy galinstan for applications in microdevices. $\mathrm{J}$ Microelectromechanical Syst 2012;21:443-450.

36. Park YL, Majidi C, Kramer R, et al. Hyperelastic pressure sensing with a liquid-embedded elastomer. J Micromech Microeng 2010;20:125029.

37. Chossat JB, Park YL, Wood RJ, et al. A soft strain sensor based on ionic and metal liquids. IEEE Sens J 2013;13: 3405-3414.

38. Adam Bilodeau R, White EL, Kramer RK. Monolithic fabrication of sensors and actuators in a soft robotic gripper. In: 2015 IEEE/RSJ International Conference on Intelligent Robots and Systems (IROS), Hamburg, Germany, September 28-October 2, 2015, pp. 2324-2329.

39. Kramer RK, Majidi C, Wood RJ. Wearable tactile keypad with stretchable artificial skin. In: Proceedings-IEEE International Conference on Robotics and Automation, Shanghai, China, May 9-13, 2011, pp. 1103-1107.

40. Kramer RK, Majidi C, Sahai R, et al. Soft curvature sensors for joint angle proprioception. In: 2011 IEEE/RSJ International Conference on Intelligent Robots and Systems, San Francisco, CA, September 25-30, 2011, pp. 19191926.

41. Vogt D, Park YL, Wood RJ. A soft multi-axis force sensor. In: Proceedings of IEEE Sensors, Taipei, Taiwan, October 28-31, 2012, pp. 1-4.

42. Case JC, White EL, Kramer RK. Soft material characterization for robotic applications. Soft Robot 2015;2:80-87.

43. Cheng S, Wu Z. Microfluidic electronics. Lab Chip 2012; 12:2782.

44. Ukkonen L, Schaffrath M, Engels DW, et al. Operability of folded microstrip patch-type tag antenna in the UHF RFID bands within 865-928 MHz. IEEE Antennas Wirel Propag Lett 2006;5:414-417. 
45. Takiguchi M, Yamada Y. Improvement of radiation efficiencies by applying folded configurations to very small meander line antennas. In: 2003 IEEE Topical Conference on Wireless Communication Technology, Honolulu, HI, October 15-17, 2003, pp. 96-97.

46. $\mathrm{Hu} \mathrm{Z}$, Cole PH, Zhang L. A method for calculating the resonant frequency of meander-line dipole antenna. In: 2009 4th IEEE Conference on Industrial Electronics and Applications, Xi'an, China, May 25-27, 2009, pp. 17831786.

47. Esa M. Electrically small High-temperature superconducting $\mathrm{Y}-\mathrm{Ba}-\mathrm{Cu}-\mathrm{O}$ meander dipole antennas for spacelimited applications. PhD thesis, University of Birmingham, 1996.

48. Balanis CA. Antenna theory: analysis and design. Electron Power 1982;28:267.

49. Antennas I, Magazine P, Politecnico V. The art of UHF RFID antenna design: impedance matching and sizereduction techniques. IEEE Antennas Propag Mag 2008;50: 66-79.

50. Abbosh A. Accurate effective permittivity calculation of printed center-fed dipoles and its application to quasi yagiuda antennas. IEEE Trans Antennas Propag 2013;61:22972300 .
51. Jackson DR, Alexopoulos NG. Analysis of planar strip geometries in a substrate-superstrate configuration. IEEE Trans Antennas Propag 1986;34:1430-1438.

Address correspondence to: Adam A. Stokes

The School of Engineering Institute for Integrated Micro and Nano Systems The University of Edinburgh The King's Buildings Edinburgh EH9 3LJ United Kingdom

E-mail: adam.stokes@ed.ac.uk

Zhirun $\mathrm{Hu}$ Microwave and Communication Systems Group School of Electrical and Electronic Engineering

University of Manchester Manchester M13 9PL United Kingdom

E-mail: z.hu@manchester.ac.uk 